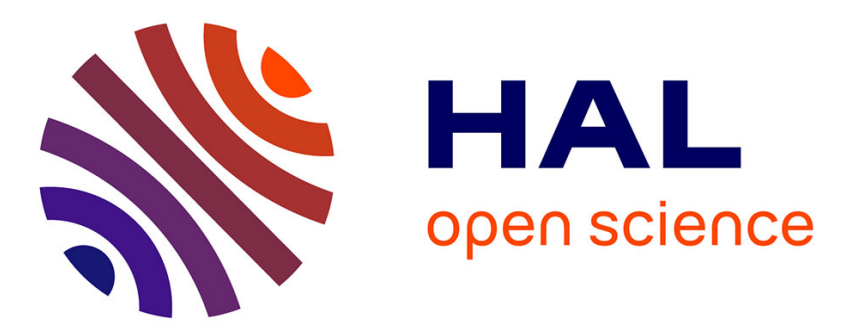

\title{
A Controller Switching between Twisting and Linear Algorithms for an Electropneumatic Actuator
} Elias Tahoumi, Franck Plestan, Malek Ghanes, Jean-Pierre Barbot

\section{To cite this version:}

Elias Tahoumi, Franck Plestan, Malek Ghanes, Jean-Pierre Barbot. A Controller Switching between Twisting and Linear Algorithms for an Electropneumatic Actuator. European Control Conference, 2018, Limassol, Cyprus. hal-01876421

\section{HAL Id: hal-01876421 https://hal.science/hal-01876421}

Submitted on 18 Sep 2018

HAL is a multi-disciplinary open access archive for the deposit and dissemination of scientific research documents, whether they are published or not. The documents may come from teaching and research institutions in France or abroad, or from public or private research centers.
L'archive ouverte pluridisciplinaire HAL, est destinée au dépôt et à la diffusion de documents scientifiques de niveau recherche, publiés ou non, émanant des établissements d'enseignement et de recherche français ou étrangers, des laboratoires publics ou privés. 


\title{
A Controller Switching between Twisting and Linear Algorithms for an Electropneumatic Actuator
}

\author{
Elias Tahoumi, Franck Plestan, Malek Ghanes and Jean-Pierre Barbot
}

\begin{abstract}
This paper presents a new control strategy for a perturbed electropneumatic actuator. This new control law is evolving between a robust second order sliding mode controller (based on twisting algorithm) and a linear one. This objective is to ensure high accuracy tracking and low energy consumption, in spite of perturbations and uncertainties. This new control solution is evaluated on an experimental set-up.
\end{abstract}

\section{INTRODUCTION}

Pneumatic actuators are very common in industrial applications due to their low cost and weight, easy installation and maintenance, and high power/weight ratio [2], [3], [7]. However, they are part of uncertain and complex systems that make this kind of actuators quite difficult to accurately control in position. Indeed, the very nature of these systems and the different interactions with external parameters and elements make them difficult to describe, model and control. Sliding mode control [4], [10], [14] has been intensively used to control this type of systems thanks to its robustness to uncertainties and perturbations.

Among the sliding mode approaches, one can cite the standard sliding mode approach [2]: the control law containing a sign function induces high frequency oscillations of the system input, a phenomenon called chattering, that engenders high frequency motions of the servodistributors' mobile parts. These high frequency motions can deteriorate the components of the actuator and therefore have to be reduced as much as possible. An alternative solution is the use of higher order sliding mode approaches (see for example [5], [7], [15]): the interest of such control laws is a better accuracy and a reduction of the chattering. However, their tuning is not a simple task given that the bounds of the uncertainties and perturbations, whose knowledge is important for the computation of the control parameters, are not easily determined and are very often overestimated. Then, another way consists in using adaptive versions (time-varying gain) of standard sliding mode controllers [9], second order sliding mode controllers [11], [13]: these approaches allow to simplify the tuning of the controller gains but transiently reduce the performances of the closed-loop system (accuracy).

A very recent solution [12] for the reduction of highfrequency oscillations consists in switching between a robust

E. Tahoumi, F. Plestan and M. Ghanes are with Ecole Centrale de Nantes, LS2N UMR CNRS 6004, 44321 Nantes , France (e-mail: elias.tahoumi@ecnantes.fr; franck.plestan@ec-nantes.fr; malek.ghanes@ec-nantes.fr).

J-P. Barbot is with ENSEA, Quartz EA 7393, 95014 Cergy-Pontoise, France (e-mail: barbot@ensea.fr). second order sliding mode controller (that is applied when the accuracy of the tracking is high relative to a predefined criteria) and linear controller (that is applied when the accuracy of the tracking is low). This approach allows to reduce chattering and energy consumption, but maintains a high level of accuracy.

The objective of the paper is to control the position of a perturbed electropneumatic actuator thanks to this novel control approach, and to show that it allows to get accurate tracking in spite of perturbation forces and uncertainties on some physical variables (especially mass flow rate). The control performances are compared to a sliding mode controller based on twisting algorithm [8] and to one based a linear state feedback, and evaluated on an experimental setup.

\section{ELECTROPNEUMATIC SYSTEM DESCRIPTION}

The electropneumatic actuator experimental setup (see Fig. 1) is composed of (for details, see [15])

- a main double acting actuator composed of two chambers $P$ and $N$. The air mass flow rate entering the chambers is controlled by 2 proportional 3-way servodistributors;

- a second actuator, identical to the main actuator, called "perturbation actuator" whose air mass flow rate is controlled by a 5-way servodistributor. Its purpose is to apply a dynamical load force on the main actuator. Note that the force control of the second actuator is performed by an analog PID controller developed by the bench manufacturer;

- a pneumatic jack which moves horizontally a load carriage of mass $M$. This carriage is coupled with the perturbation actuator.

The mathematical model of the electropneumatic system (detailed in [15]) reads as

$$
\begin{aligned}
\dot{p}_{P} & =\frac{k r T}{V_{P}(y)}\left[\varphi_{P}+\psi_{P} \cdot u-\frac{S}{r T} p_{P} v\right] \\
\dot{p}_{N} & =\frac{k r T}{V_{P}(y)}\left[\varphi_{N}-\psi_{N} \cdot u+\frac{S}{r T} p_{N} v\right] \\
\dot{v} & =\frac{1}{M}\left[S\left(p_{P}-p_{N}\right)-b_{v} v-F_{\text {ext }}\right] \\
\dot{y} & =v
\end{aligned}
$$


This model can be written as a nonlinear system affine in the control input, i.e.

$$
\dot{x}=f(x)+g(x) u
$$

with $x$ the state vector defined as $x=\left[\begin{array}{llll}p_{N} & p_{P} & v & y\end{array}\right]^{T} \in$ $\mathcal{X} \subset \mathbb{R}^{4}$, and $u$ the control input. Furthermore, vectors $f(x)$ and $g(x)$ read as

$$
\begin{aligned}
f(x)= & {\left[\begin{array}{c}
\frac{k r T}{V_{P}(y)}\left[\varphi_{P}-\frac{S}{r T} p_{P} v\right] \\
\frac{k r T}{V_{P}(y)}\left[\varphi_{N}+\frac{S}{r T} p_{N} v\right] \\
\frac{1}{M}\left[S\left(p_{P}-p_{N}\right)-b_{v} v-F_{\mathrm{ext}}\right] \\
v
\end{array}\right] } \\
g(x)= & {\left[\begin{array}{c}
\frac{k r T}{V_{P}(y)} \psi_{P} \\
-\frac{k r T}{V_{N}(y)} \psi_{N} \\
0 \\
0
\end{array}\right] }
\end{aligned}
$$

with $y$ the piston position, $v$ its velocity, $F_{\text {ext }}$ the external perturbation produced by the perturbation actuator, $p_{P}$ and $p_{N}$ the pressures in chambers $P$ and $N$ respectively, $r$ the perfect gas constant, $b_{v}$ the viscous friction coefficient, $T$ the supply temperature, $k$ the polytropic constant, and $S$ the piston surface. $u$ is the control input of the system; physically, this input is a voltage acting on both servodistributors: given that there are two servodistributors, a voltage is applied to the first one whereas the opposite value is applied to the other one. The volume in each chamber reads as

$$
\begin{aligned}
& V_{P}(y)=V_{0}+S \cdot y \\
& V_{N}(y)=V_{0}-S \cdot y
\end{aligned}
$$

with $V_{0}$ the half-cylinder volume. The functions $\varphi_{P}, \varphi_{N}$, $\psi_{P}$ and $\psi_{N}$ describe the mass flow rate and defined as $5^{\text {th }}$ order polynomials of the pressures as depicted in [11]. $\mathcal{X}$ is the operating domain such that

$$
\begin{gathered}
\mathcal{X}=\left\{x \mid 1 \text { bar } \leq p_{N} \leq 7 \text { bar, } 1 \text { bar } \leq p_{P} \leq 7\right. \text { bar } \\
\left.|y| \leq 72 \mathrm{~mm},|v| \leq 1 \mathrm{~m} . \mathrm{s}^{-1}\right\}
\end{gathered}
$$

Mechanical and physical parameters of the main actuator read as

$$
\begin{aligned}
& M=3.4 \mathrm{~kg}, V_{0}=3.40 \cdot 10^{-4} \mathrm{~m}^{3}, S=0.0045 \mathrm{~m}^{2}, \\
& b_{v}=50, k=1.2, r=287 \mathrm{~J} . \mathrm{kg}^{-1} \cdot \mathrm{K}^{-1}, T=293^{\circ} \mathrm{K} .
\end{aligned}
$$

\section{CONTROLler DESIGN}

\section{A. Problem statement}

The control objective is to force the main actuator to track a sufficiently differentiable reference signal $y_{\text {ref }}$. Given that the controller developed in the sequel is based on the sliding mode concept, define the sliding variable

$$
\sigma=v-\dot{y}_{\text {ref }}+\lambda\left(y-y_{\text {ref }}\right)
$$

with $\lambda>0$. One recalls from the sliding mode theory [14], [10] that the control law has to ensure at least ${ }^{1} \sigma=0$ in a finite time; such a behavior is called sliding mode. Once the sliding mode is established, and given the definition of $\sigma$, it yields that the tracking error $e_{y}=y-y_{\text {ref }}$ converges to 0 exponentially with a rate depending on $\lambda$.

\section{B. Control scheme}

The relative degree ${ }^{2}$ of $\sigma$ with respect to $u$ is $2 ; \ddot{\sigma}$ dynamics read as

$$
\begin{aligned}
\ddot{\sigma}= & \frac{1}{M}\left[S\left(\dot{p}_{P}-\dot{p}_{N}\right)-b_{v} \dot{v}-\dot{F}_{\mathrm{ext}}\right]-y_{\mathrm{ref}}^{(3)} \\
& +\frac{\lambda}{M}\left[S\left(p_{p}-p_{N}\right)-b_{v} v-F_{\mathrm{ext}}\right]-\ddot{y}_{\mathrm{ref}} \\
= & \Psi(.)+\Phi(.) u
\end{aligned}
$$

with

$$
\begin{aligned}
\Psi(.)= & \frac{S k r T}{M}\left[\frac{\varphi_{P}}{V_{P}(y)}-\frac{\varphi_{N}}{V_{N}(y)}\right]-\frac{1}{M}\left(\dot{F}_{\mathrm{ext}}+M y_{\text {ref }}^{(3)}\right) \\
& -\frac{S^{2} k v}{M}\left[\frac{P_{p}}{V_{P}(y)}+\frac{P_{N}}{V_{N}(y)}\right]-\lambda \ddot{y}_{r e f} \\
& +\frac{M \lambda-b_{v}}{M^{2}}\left[S\left(p_{P}-p_{N}\right)-b_{v} v-F_{\mathrm{ext}}\right] \\
\Phi(.)= & \frac{S k r T}{M}\left[\frac{\psi_{P}}{V_{P}(y)}+\frac{\psi_{N}}{V_{N}(y)}\right]
\end{aligned}
$$

Functions $\Psi($.$) and \Phi($.$) are uncertain functions due to$ parametric uncertainties (for example, the temperature $T$ in the chamber is time-varying whereas it is supposed to be constant in the control design) and given that there are external perturbations $F_{\text {ext }}$ (consequently $\dot{F}_{\text {ext }}$ ) applied by the perturbation actuator on the main one. Then, the functions $\Psi($.$) and \Phi($.$) can be written as$

$$
\begin{aligned}
& \Psi(.)=\Psi_{\mathrm{Nom}}(.)+\Delta \Psi(.) \\
& \Phi(.)=\Phi_{\mathrm{Nom}}(.)+\Delta \Phi(.)
\end{aligned}
$$

with $\Psi_{\text {Nom }}($.$) and \Phi_{\text {Nom }}($.$) being the nominal terms and$ $\Delta \Psi($.$) and \Delta \Phi($.$) the uncertain terms. In [13], it has$ been numerically observed that, under current operating conditions, $\Psi_{\text {Nom }}$ and $\Phi_{\text {Nom }}$ are bounded and only depend on measured or estimated variables. Furthermore, $\Phi_{\text {Nom }}>$ $0 \forall x \in \mathcal{X}$. Hence, a control law is given as

$$
u=\frac{1}{\Phi_{\mathrm{Nom}}}\left(-\Psi_{\mathrm{Nom}}+\omega\right)
$$

\footnotetext{
${ }^{1}$ If the control law is a standard (first order) sliding mode one, the system trajectories are forced such that $\sigma=0$ in a finite time; if the control law is based on high order sliding mode approach, a finite number (depending on sliding mode order) of time derivatives of $\sigma$ are also forced to 0 in a finite time.

${ }^{2}$ Given that the relative degree is lower than the state dimension, it means that there is internal dynamics incorporating the pressures in chambers $P$ and $N$. It is supposed that these internal dynamics are stable in the operating domain.
} 

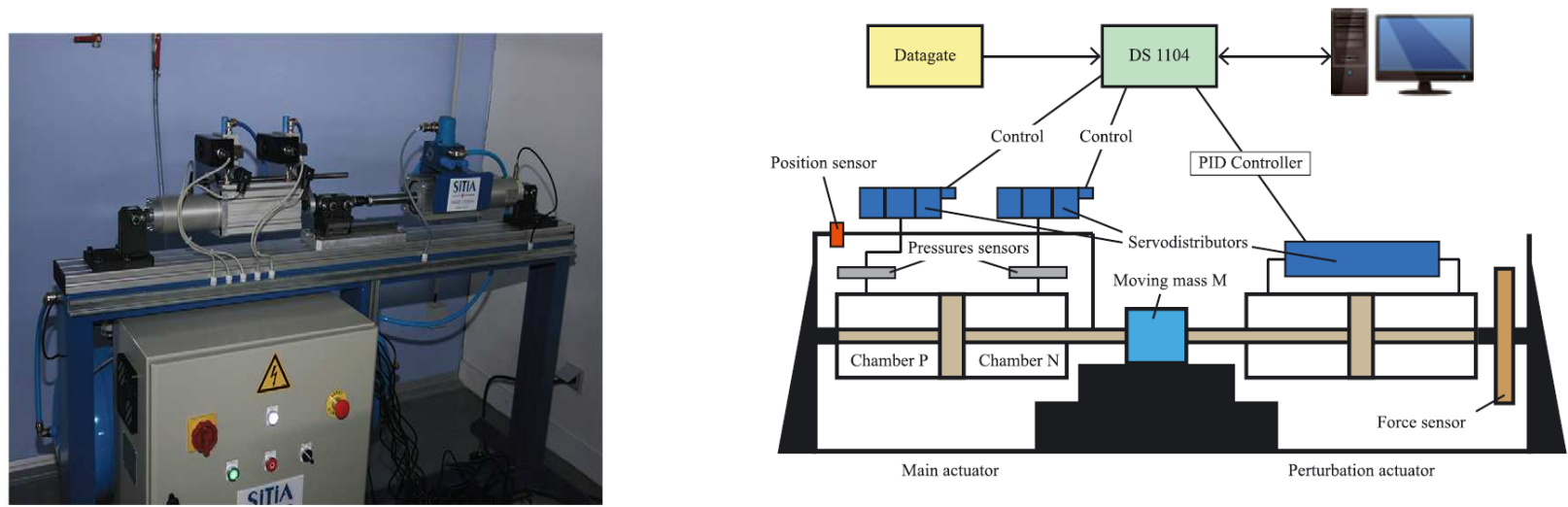

Fig. 1. Left. Photo of the electropneumatic setup. Right. Scheme of the control architecture of the electropneumatic setup [15]

with $\omega$ being a controller switching between the twisting and the linear algorithms (defined in the next subsection). After substituting (4)-(5) in (3), one gets

$$
\begin{aligned}
\ddot{\sigma} & =\Delta \Psi-\Delta \Phi \Phi_{\mathrm{Nom}}^{-1} \Psi_{\mathrm{Nom}}+\left(1+\Delta \Phi \Phi_{\mathrm{Nom}}^{-1}\right) \cdot \omega \\
& =a(x, t)+b(x, t) \cdot \omega
\end{aligned}
$$

with

$$
\begin{aligned}
& a(x, t)=\Delta \Psi-\Delta \Phi \Phi_{\text {Nom }}^{-1} \Psi_{\text {Nom }} \\
& b(x, t)=1+\Delta \Phi \Phi_{\text {Nom }}^{-1}
\end{aligned}
$$

As previously mentioned, under the considered operating conditions, the functions $\Psi$ and $\Phi$ are bounded with $\Phi_{\text {Nom }}>$ 0 . Therefore, there exist positive constants $a_{M}, b_{m}$ and $b_{M}$ such that, $\forall x \in \mathcal{X}$ and $t \geq 0$,

$$
\begin{aligned}
& |a(x, t)| \leq a_{M} \\
& 0<b_{m} \leq b(x, t) \leq b_{M} .
\end{aligned}
$$

\section{Control law $\omega$}

The control law $\omega$ is given in order to stabilize system (6) in spite of uncertainties and perturbations while reducing the energy consumption. Both objectives can be fulfilled by the control strategy proposed in [12] that reads as

$$
\omega=-k_{1}|\sigma|^{\frac{\alpha(t)}{2-\alpha(t)}} \operatorname{sign}(\sigma)-k_{2}|\dot{\sigma}|^{\alpha(t)} \operatorname{sign}(\dot{\sigma}),
$$

where $k_{1}$ and $k_{2}$ are the controller gains. The time-varying exponent $\alpha$ is switching between 0 and 1 thanks to the following law $^{3}$

$$
\alpha= \begin{cases}1 & \text { if }|\sigma|<\varepsilon_{\sigma} \wedge|\dot{\sigma}|<\varepsilon_{\dot{\sigma}} \\ 0 & \text { otherwise }\end{cases}
$$

Parameters $\varepsilon_{\sigma}$ and $\varepsilon_{\dot{\sigma}}$ are positive constants set by the user. Note that $\alpha=0$ corresponds to the twisting algorithm [8] and $\alpha=1$ means that a linear state feedback is applied.

\section{Main ideas.}

- The controller (8) is based on a homogeneous control law [1] where the exponent $\alpha$ is considered constant.

\footnotetext{
${ }^{3}$ The notation $\wedge$ is used for the logical AND operator.
}

This form of controller will give all its interest for a continuously time-varying exponent (see Section V);

- The controller (8)-(9) switches from a twisting controller $(\alpha=0)$ to a linear one $(\alpha=1)$, the commutation depends on the accuracy performance of the closed-loop system. The accuracy depends on both parameters $\epsilon_{\sigma}$ and $\epsilon_{\dot{\sigma}}$;

- The twisting algorithm $(\alpha=0)$ is known for its high accuracy and robustness with respect to matching perturbations; however, it is high energy consuming. Then, the proposed control scheme allows the use of the twisting algorithm only when the desired accuracy is not established. In this case, as a second order sliding mode algorithm, the twisting forces the system trajectories to reach a vicinity of the origin of the phase plan $(\sigma, \dot{\sigma})^{4}$.

- Once the trajectories have reached a vicinity of the origin of the phase plan $(\sigma, \dot{\sigma})$, the idea is to reduce the energy consumption. Then, as long as the system trajectories are evolving in the origin vicinity defined via $\epsilon_{\sigma}$ and $\epsilon_{\dot{\sigma}}$, the linear controller $(\alpha=1)$ is applied.

- Given that the linear controller is less robust in case of perturbations, the system trajectories can leave the origin vicinity. In this case, the parameter $\alpha$ switches to 0 , the twisting algorithm is applied, and so on.

The controller (8)-(9) allows a trade-off between the twisting algorithm and the linear state feedback and has the advantages of the twisting algorithm (robustness) and linear state feedback (low energy consumption) with their drawbacks reduced.

To summarize, the logic of controller (8)-(9) is as follows. If the trajectories of system (6) are inside $\mathcal{D}$ such that

$$
\mathcal{D}=\left\{(\sigma, \dot{\sigma})|| \sigma\left|<\varepsilon_{\sigma} \wedge\right| \dot{\sigma} \mid<\varepsilon_{\dot{\sigma}}\right\}
$$

then the desired accuracy of the system is reached; therefore, the linear state feedback $(\alpha=1)$ is applied to decrease the

\footnotetext{
${ }^{4}$ This property is connected to the concept of real sliding mode [10]: as a second order sliding mode control, in case of sampled control input with $\tau$ the sampling period, the twisting control law allows the system trajectories to reach the so-called real sliding mode set defined by $|\sigma| \leq \mu_{1} \tau^{2}$ and $|\dot{\sigma}| \leq \mu_{2} \tau$.
} 
energy consumption. If the trajectories of the system are outside $\mathcal{D}$, it means that the desired accuracy is not reached probably due to perturbations and uncertainties. Hence, the twisting algorithm $(\alpha=0)$ is applied in order to force the trajectories back to $\mathcal{D}$.

Theorem 1 ([12]): Consider system (6) with the control strategy (8)-(9). Suppose that the gains $k_{1}, k_{2}$ and the parameters $\varepsilon_{\sigma}$ and $\varepsilon_{\dot{\sigma}}$ satisfy the conditions

$$
\begin{gathered}
k_{1}>k_{2}>0, \quad\left(k_{1}-k_{2}\right) b_{m}>a_{M}, \\
\left(k_{1}+k_{2}\right) b_{m}-a_{M}>\left(k_{1}-k_{2}\right) b_{M}+a_{M}, \\
k_{1} \varepsilon_{\sigma}+k_{2} \varepsilon_{\dot{\sigma}}<k_{1}-k_{2},
\end{gathered}
$$

then $\sigma$ and $\dot{\sigma}$ are evolving in a finite time in a vicinity of the origin defined as

$$
\begin{aligned}
& |\sigma|<\frac{\varepsilon_{\dot{\sigma}}^{2}}{2 K_{M}^{\min }}+\varepsilon_{\sigma} \\
& |\dot{\sigma}|<\sqrt{\varepsilon_{\dot{\sigma}}^{2}+2 K_{m}^{\max \varepsilon_{\sigma}}}
\end{aligned}
$$

with $K_{m}^{\max }=b_{M}\left(k_{1}-k_{2}\right)+a_{M}$ and $K_{M}^{\min }=b_{m}\left(k_{1}+\right.$ $\left.k_{2}\right)-a_{M}$.

Details of the proof can be found in [12].

Remark 1: Condition (11) is needed to guarantee the convergence of the closed loop system when the twisting algorithm is applied [8] whereas condition (12) is required to make sure that controller (8)-(9) is less energy consuming than the twisting algorithm [12].

\section{EXPERIMENTAL RESULTS}

\section{A. Context}

Controller (8)-(9) is now implemented on the experimental setup using MATLAB/Simulink coupled with dSpace DS1104 datacard. The sampling period has been set to $\varsigma=0.2 \mathrm{~ms}$. The position of the mass load as well as the pressures $p_{P}$ and $p_{N}$ are measured via sensors. The velocity and the acceleration are obtained using a differentiator.

Subsequently, the performances of the three controllers (the linear one, the twisting one, and the controller (8)-(9)) are analyzed. This analysis is made by evaluating the tracking error with respect to a position reference trajectory $y_{\text {ref }}$ detailed in the sequel, and by evaluating the energy consumption (from a control input point of view).

The mass load connected to the main actuator has to track a reference trajectory that reads as

$$
\begin{array}{ll}
y_{\text {ref }}=0.04 \sin (0.15 \pi t+\pi) & \text { for } \quad 0 \leq t<20 \mathrm{~s} \\
y_{\text {ref }}=0 & \text { for } \quad 20 \leq t<40 \mathrm{~s} \\
y_{\text {ref }}=0.04 \sin (0.3 \pi t) & \text { for } \quad 40 \leq t \leq 60 \mathrm{~s}
\end{array}
$$

This reference trajectory has been used in order to evaluate the performances of the controllers in case of relatively fast and slow motions (sinusoidal references) or in case of constant position. All the tests are made by considering perturbations. Thus, the selected reference trajectory for the perturbation actuator is $F_{\text {ext }}=500 \sin (0.34 \pi t)(N)$ (Fig. 2). This trajectory is tracked using a PID controller parametrized by the setup manufacturer. Note that the perturbation is considered unknown by the controller (only the bound of the perturbation is known).

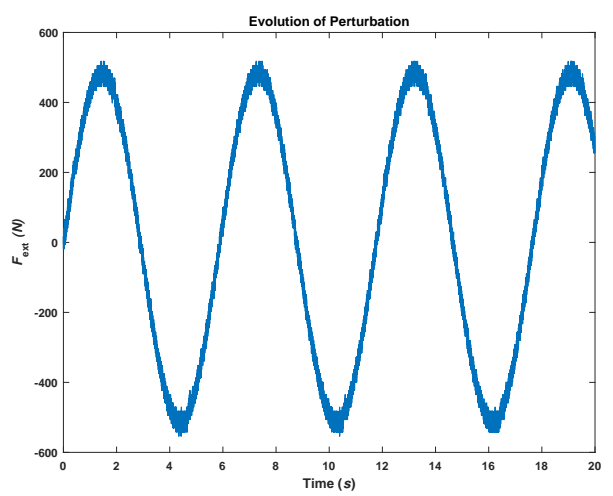

Fig. 2. External perturbation $F_{\text {ext }}(N)$ versus time $(s)$.

The gains $k_{1}$ and $k_{2}$ of the three controllers are set to 3000 and 1500 respectively. The parameters $\varepsilon_{\sigma}$ and $\varepsilon_{\dot{\sigma}}$ of (8)(9) are taken 0.01 and 0.9 respectively. These parameters satisfy conditions (11)-(12); furthermore, this choice has been made in order to get the best performances during the experimentation, for all controllers. Finally, the performances of the controllers are analyzed by evaluating the mean of the absolute value of the tracking accuracy $e_{y}=y-y_{\text {ref }}$ all along the trajectories. Furthermore, the energy consumption will be evaluated via two values; first-of-all, the standard deviation of the control input $u$ which is a good indicator of the presence (or not) of chattering that is high energy consuming. Another indicator of the energy consumption from instant $t_{0}$ to $t_{1}$ is defined by

$$
\mathcal{E}=\int_{t_{0}}^{t_{1}} u^{2} d t
$$

\section{B. Results}

As it can be seen from Fig. 3, both controller (8)-(9) and the twisting algorithm lead to a good tracking of the reference trajectory whereas the linear state feedback has a low tracking accuracy. The mean tracking accuracy provided by controller (8)-(9) is significantly better than that of the linear state feedback (the mean tracking error is divided by 34) and comparable to that of the twisting algorithm (see Fig. 4 and Table I).

The consumed energy $\mathcal{E}$ (evaluated for $0 \leq t \leq 60 \mathrm{~s}$ ) by controller (8)-(9) is less than the consumed energy with the twisting algorithm $(60.16 \%$ decrease in energy consumption) but greater than the energy consumed with the linear state feedback (see Fig. 5 and Table I).

The evolution of $\alpha$ with controller (8)-(9) for $22.5 \leq t \leq 23 \mathrm{~s}$ is depicted in Fig. 6. The mean value of $\alpha$ is 0.67 ; this means that the linear state feedback is applied $67 \%$ of the time; therefore, the chattering effect with controller (8)-(9) is reduced but the accuracy is kept at a very high level (see 

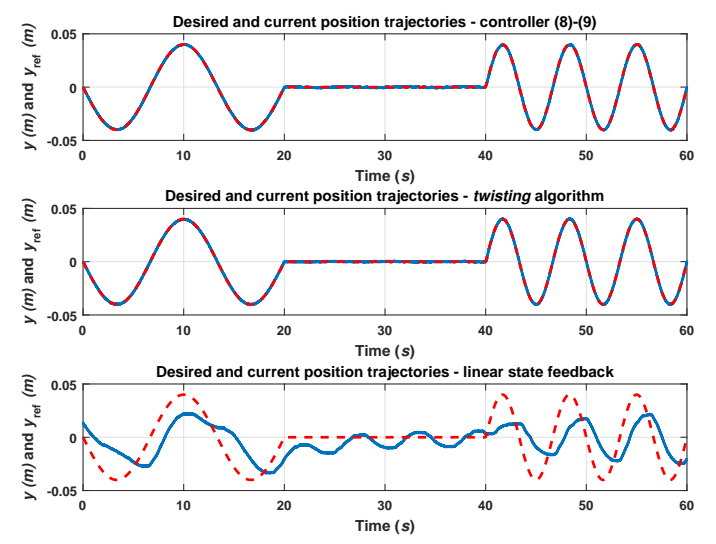

Fig. 3. Reference position $y_{\text {ref }}(m)$ (red dotted) and measured position $y(m)$ (blue solid) versus time $(s)$ for (from top to bottom) controller (8)-(9), twisting algorithm and linear state feedback.
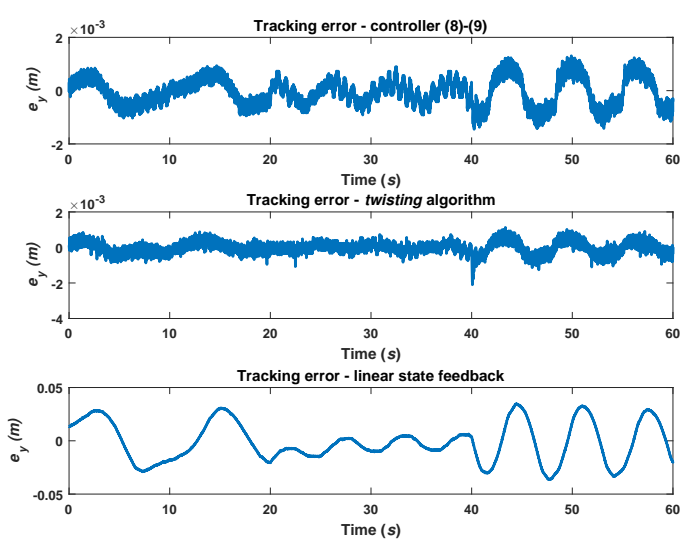

Fig. 4. Tracking error $e_{y}(m)$ versus time $(s)$ for (from top to bottom) controller (8)-(9), twisting algorithm and linear state feedback.

\begin{tabular}{|c|c|c|c|}
\cline { 2 - 4 } \multicolumn{1}{c|}{} & $\begin{array}{c}\text { Controller } \\
(8)-(9)\end{array}$ & $\begin{array}{c}\text { Twisting } \\
\text { Algorithm }(\alpha=0)\end{array}$ & $\begin{array}{c}\text { Linear State } \\
\text { Feedback }(\alpha=1)\end{array}$ \\
\hline Energy $\mathcal{E}$ & 164.02 & 411.68 & 28.06 \\
\hline $\operatorname{Mean}\left(\left|e_{y}\right|\right)$ & $4.39 \cdot 10^{-4}$ & $2.46 \cdot 10^{-4}$ & $1.52 \cdot 10^{-2}$ \\
\hline $\operatorname{Std}(u)$ & 1.65 & 2.62 & 0.68 \\
\hline $\operatorname{Mean}(\alpha)$ & 0.67 & \multicolumn{3}{c}{} \\
\cline { 2 - 4 } & \multicolumn{2}{|c}{} &
\end{tabular}

TABLE I

Energy $\mathcal{E}$, Mean $\left(\left|e_{y}\right|\right), S t d(u)$ AND $M e a n(\alpha)$ FOR CONTROLLER (8)-(9), twisting ALGORITHM AND LINEAR STATE FEEDBACK FOR

$$
0 \leq t \leq 60 s
$$

Table I). The indicator used to quantify the reduction of the chattering is the standard deviation (Std) of the control input $u$ : it can be seen that, thanks to the proposed controller, the standard deviation of $u$ is less than that of the twisting algorithm (-37.02\% - see Table I).

All these tests and analysis allow to conclude that the proposed controller has kept the advantages of the linear and twisting controllers but with a strong reduction of their respective drawbacks. Thus, one gets a robust and accurate control law, with reduced chattering and energy consumption.
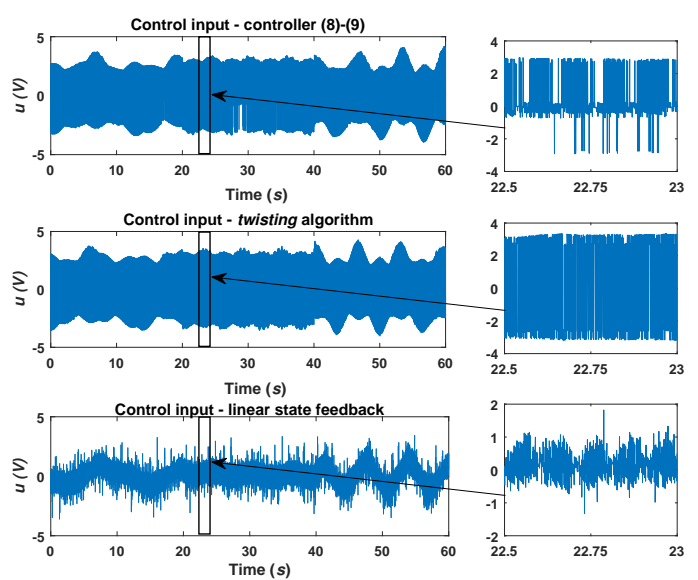

Fig. 5. Control input $u(V)$ versus time $(s)$ for (from top to bottom) controller (8)-(9), twisting algorithm and linear state feedback.

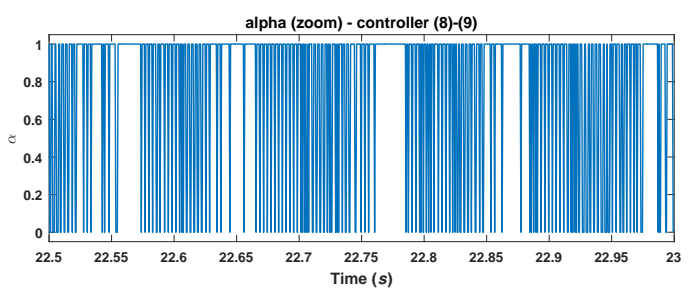

Fig. 6. Exponent $\alpha$ versus time $(s)$ for $22.5 \leq t \leq 23 s$.

\section{Prospective towards CONTINUOUS $\alpha$-DYNAmics}

Another solution ${ }^{5}$ consists in proposing a controller (8) for which the exponent $\alpha$ dynamically (continuously) evolves between 0 and 1 . It is necessary to recall that the homogeneous controller (8) is intrinsically robust even if $\alpha$ is not strictly equal to 0 ; in fact, it is not necessary to have $\alpha=0$ (twisting algorithm) to have robust features. Furthermore, when $\alpha \neq 0$, the control law is smoother and then, there is a natural reduction of the chattering, and therefore a reduction of energy consumption.

Then, the idea is the following: the value of $\alpha$ decreases dynamically when the accuracy of the system is low, in order to get if necessary the twisting algorithm. It is important to note that it could be unnecessary to apply the twisting algorithm $(\alpha=0)$ to achieve the required accuracy. When this latter is achieved, the value of the exponent $\alpha$ is increased in order to decrease the energy consumption.

\section{A. A continuous adaptation law for $\alpha$}

In the sequel, the following adaptation law for $\alpha$ in the frame of the control law (8) has been chosen as (of course, this choice is not unique)

$$
\begin{aligned}
& \dot{\alpha}= \begin{cases}-1 & \text { if } \beta>0 \wedge \alpha \geq 1 \\
1 & \text { if } \beta<0 \wedge \alpha \leq 0, \quad \alpha(0)=0 \\
\beta & \text { otherwise }\end{cases} \\
& \beta=k\left(-1+\operatorname{sign}\left(\varepsilon_{\sigma}-|\sigma|\right)+\operatorname{sign}\left(\varepsilon_{\dot{\sigma}}-|\dot{\sigma}|\right)\right)
\end{aligned}
$$

${ }^{5}$ The use of a continuous adaptation law for $\alpha$ is presented here as a prospective and will be theoretically studied in future works. 
with $k, \varepsilon_{\sigma}$ and $\varepsilon_{\dot{\sigma}}$ positive constants.

The minimum/maximum values of the output of the integrator of $\dot{\alpha}$ are limited to respectively $0 / 1$. This is necessary to ensure the trade-off between the linear and twisting controllers; therefore, the gains $k_{1}$ and $k_{2}$ have to be tuned to ensure the stability of the closed-loop system $\forall \alpha \in$ $[0,1]$ fixed. Hence, the gains should satisfy (11) (note that exponential stability of the closed-loop system with the linear state feedback is ensured if the gains are positive [6], that is a condition guaranteed by (11)).

\section{Main ideas.}

- At $t=0, \alpha$ is initialized at 0: the twisting algorithm is applied to ensure the convergence of the system trajectories, in a finite time, to a vicinity $\mathcal{D}$ of the origin of the phase plan $(\sigma, \dot{\sigma})$;

- Once $(\sigma, \dot{\sigma}) \in \mathcal{D}$, it means that desired accuracy is reached. Then, $\dot{\alpha}=k$ : as a consequence, $\alpha$ increases towards 1 to reduce the energy consumption;

- If $\sigma$ or $\dot{\sigma}$ is outside $]-\varepsilon_{\sigma}, \varepsilon_{\sigma}$ [ or ] $-\varepsilon_{\dot{\sigma}}, \varepsilon_{\dot{\sigma}}$ [ respectively, then it means that desired accuracy is not reached; hence, $\dot{\alpha}=-k$ : $\alpha$ decreases in order to increase the accuracy, and so on;

- if both variables $\sigma$ and $\dot{\sigma}$ are outside of their respective intervals, then the rate by which $\alpha$ decreases towards zero is three times faster $(\dot{\alpha}=-3 k)$ to restore the accuracy faster.

\section{B. Experimental results}

The controller (8)-(15) is applied to the experimental setup with the following parameters

$$
k=15, k_{1}=3000, k_{2}=1500, \varepsilon_{\sigma}=0.01, \varepsilon_{\dot{\sigma}}=0.7
$$

The results are shown in Fig. 7. The average tracking error is $4.83 \cdot 10^{-4}$, that corresponds to $96.82 \%$ decrease with respect to the linear state feedback. The energy consumed for $0 \leq t \leq 60 \mathrm{~s}$ is $137.16,66.68 \%$ decrease from the twisting algorithm. The average value of $\alpha$ is 0.38 . The nonapplication of the twisting algorithm has a great interest, as proved by the standard deviation of $u$, that is equal to 1.51 , a $42.37 \%$ decrease with respect to the twisting algorithm. As a consequence, it means that the chattering effect is reduced, the control input has a better profile for the actuator, whereas the accuracy performance is very good.

\section{CONCLUSION}

A new controller for an electropneumatic actuator has been developed combining the advantages of the linear state feedback and twisting controller. The accuracy of the system is taken into account in order to switch between the twisting and linear algorithms thanks to the adaptation of a parameter (exponent) appearing in the controller. Hence, an accurate and low energy consuming controller is obtained. Experimental results show the effectiveness of the controllers, with two different exponent adaptation laws.

Future works will be dedicated to further analysis of the
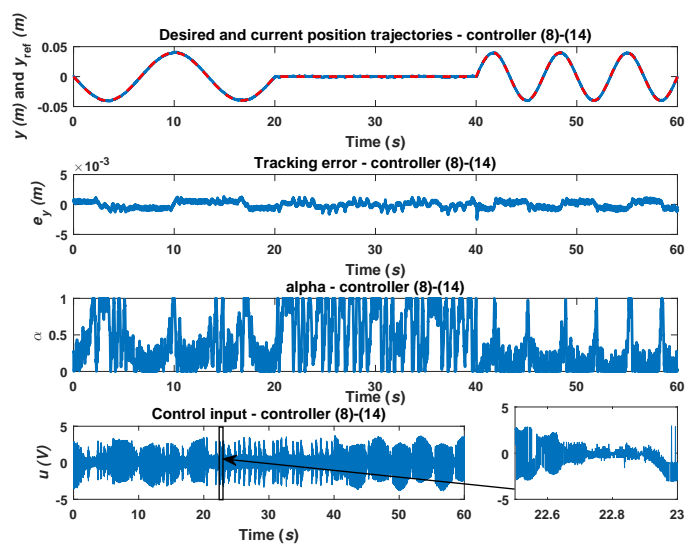

Fig. 7. From top to bottom: reference position $y_{\text {ref }}(m)$ (red dotted) and measured position $y(m)$ (blue solid), tracking error $e_{y}(m)$, control input $u(V)$ and exponent $\alpha$ versus time $(s)$ for controller (8)-(15).

control solution with the continuous exponent adaptation law, and an estimation of its domain of convergence.

\section{REFERENCES}

[1] S.P. Bhat and D.S. Bernstein. "Finite-time stability of homogeneous systems." In American Control Conference, Albuquerque, New Mexico, USA, 1997.

[2] M. Bouri and D. Thomasset, "Sliding control of an electropneumatic actuator using an integral switching surface," IEEE Transactions on Control Systems Technology, vol. 9, no. 2, pp. 368-375, 2001.

[3] X. Brun, S. Sesmat, D. Thomasset, and S. Scavarda, "A comparative study between two control laws of an electropneumatic actuator," in European Control Conference, ECC, Karlsruhe, Germany, 1999, pp. 2967-2974.

[4] C. Edwards and S. Spurgeon, Sliding mode control: theory and applications. Taylor and Francis, London, UK, 1998.

[5] A. Estrada and F. Plestan, "Second order sliding mode output feedback control with switching gains application to the control of a pneumatic actuator," Journal of the Franklin Institute, vol. 351, no. 4, pp. 2335 2355, 2014.

[6] M. Gopal. Control systems: principles and design. Tata McGraw-Hill Education, 2002.

[7] S. Laghrouche, M. Smaoui, F. Plestan, and X. Brun, "Higher order sliding mode control based on optimal approach of an electropneumatic actuator," International Journal of Control, vol. 79, no. 2, pp. 119-131, 2006

[8] A. Levant, "Sliding order and sliding accuracy in sliding mode control," International journal of control, vol. 58, no. 6, pp. 1247 1263, 1993.

[9] F. Plestan, Y. Shtessel, V. Bregeault, and A. Poznyak, "Sliding mode control with gain adaptation application to an electropneumatic actuator" Control Engineering Practice, vol. 21, no. 5, pp. 679-688, 2013.

[10] Y. Shtessel, C. Edwards, L. Fridman, and A. Levant, Sliding mode control and observation. Springer, New York, USA, 2014.

[11] Y. Shtessel, M. Taleb, and F. Plestan, "A novel adaptive-gain supertwisting sliding mode controller: Methodology and application," Automatica, vol. 48, no. 5, pp. 759-769, 2012.

[12] E. Tahoumi, M. Ghanès, F. Plestan, and J.-P. Barbot, "A new controller switching between linear and twisting algorithms", In American Control Conference, Milwaukee, Wisconsin, USA, 2018.

[13] M. Taleb, A. Levant, and F. Plestan, "Pneumatic actuator control: Solution based on adaptive twisting and experimentation," Control Engineering Practice, vol. 21, no. 5, pp. 727-736, 2013.

[14] V. I. Utkin, Sliding modes in control and optimization. Springer, 1992.

[15] X. Yan, F. Plestan, and M. Primot, "A new third-order sliding mode controller-application to an electropneumatic actuator," IEEE Transactions on Control Systems Technology, vol. 25, no. 2, pp. 744751,2017 